\title{
Hypertension status among the adult population in a rural community of Keraniganj Upazila, Dhaka, Bangladesh
}

\author{
A Farhana ${ }^{1 凶}$, M Shakil $^{2}$, BU Khan ${ }^{3}$, MS Laskar $^{4}$, M Alam $^{5}$
}

\begin{abstract}
Hypertension or high blood pressure is a growing public health concern among the Bangladeshi adult population. The objective of this cross-sectional descriptive type of study was to estimate the proportion of hypertension in an adult population of Keraniganj Upazila, Dhaka. The sample size was 199 selected purposively. Among them, 53.8\% were female, 82.4\% Muslims. Of the total respondents, $15.6 \%$ were hypertensive and highest proportion (29.0\%) of hypertensive patients was found in the age group of $\geq 50-<60$ years. The study revealed that people who took extra fatty food for 3-5 days per week, people who took extra salt in their food, respondents had a positive family history of hypertension or diabetes mellitus were more prone to develop hypertension. The study also revealed that smokers were in higher risk of development of hypertension than the non-smokers were and the rate of hypertension was $36.8 \%$ among those having higher waist circumference within $100 \mathrm{~cm}-109 \mathrm{~cm}$ than those having lower waist circumference within $60 \mathrm{~cm}-69 \mathrm{~cm}$. Newly identified hypertensive cases were $7.0 \%$ of the study population. Results of this study have profound implications for hypertension prevention programs in the rural community, pointing out the need for increased focus in addressing hypertension along with its all background causes.
\end{abstract}

Keywords: hypertension, risk factors, adult population, rural community.

\section{Introduction}

Hypertension is a significant public health challenge, which affects approximately one billion people worldwide. ${ }^{1}$ According to the World Health Organization, hypertension is the leading risk factor for mortality $(12.7 \%$ of deaths attributable) followed by tobacco use $(8.7 \%)$ and high blood glucose $(5.8 \%){ }^{2}$ Each year at least 7.1 million people die as a consequence of hypertension. ${ }^{3}$ The overall average prevalence of hypertension in the world was estimated at $35.0 \%(37.0 \%$ in men and $31.0 \%$ in women). ${ }^{4}$ Hypertension has become a significant problem, is already established in high-income countries, and also emerging in many low- and middleincome countries experiencing an epidemiological transition from communicable to noncommunicable chronic disease. Hypertension also has a major impact on healthcare costs, contributing to around $10.0 \%$ of total healthcare spending globally.

In most developing countries, including Bangladesh, hypertension often remains undiagnosed and untreated and, even when

\footnotetext{
1. A Farhana, MBBS, MPH, MPhil, Associate Professor of Community Medicine, MH Samorita Medical College, Dhaka.Email: famin1975@gmail.com

2. M Shakil, MBBS, MPH, Associate Professor of Community Medicine, Army Medical College, Comilla

3. BU Khan, MBBS, FCPS, MRCS, Associate Professor of Surgery, Holy Family Red Crescent Medical College, Dhaka

4. MS Laskar, MD, MPH, PhD, Professor of Community Medicine, Gazi Medical College, Khulna

5. M Alam, MBBS, MPH, MPhil, Professor of Community Medicine, MH Samorita Medical College, Dhaka
} 
treated, a large proportion still has uncontrolled blood pressure (BP). Despite effective therapies and lifestyle interventions, adequate control of hypertension remains a challenge. $^{5}$

Modern lifestyle factors are responsible for a growing burden of hypertension: physical inactivity, salt-rich diets with processed and fatty foods and alcohol and tobacco use. High BP can also be secondary to other conditions- kidney disease, for example. ${ }^{6}$ There are general risk factors that can be responsible for raising anyone's risk of hypertension, however. These include obesity, age, sex, and certain lifestyles such as greater uptakes of dietary salt, excessive alcohol, low dietary potassium intake and physical inactivity. Other risk factors are a family history of the disease and chronic stress. ${ }^{7}$ So, lifestyle changes are important for both treatment and prevention of high BP, and they can be as effective as a drug treatment. The added advantage is that there are wider effects on heart health.

In recent years, hypertension and cerebrovascular diseases have increased in SouthEast Asia including Bangladesh because of rapid urbanization, increased life expectancy, unhealthy diet, and lifestyle changes. The Bangladesh Non-communicable Diseases Risk Factor Survey in 2010 estimated the prevalence of hypertension among adults between 16.0-20.0\%. In addition, the Bangladesh Health, Nutrition and Demographic Survey in 2011 reported the prevalence of hypertension among adults $34.0 \%{ }^{8}$ Furthermore, a meta-analysis of studies between 1995 and 2010 found the pooled prevalence of hypertension to be $13.7 \%$, with an increasing trend and higher rate in urban versus rural areas (22.2\% vs $14.3 \%$, respectively). ${ }^{5}$

An epidemiological shift has been observed in the prevalence of hypertension in developing countries as compared to developed countries. Studies from India and Bangladesh have shown an upward trend in the prevalence of hypertension. The prevalence of hypertension has increased by 30 times among the urban population over 55 years and about 10 times among rural population over 36 years. $^{9}$ Developing countries are increasingly faced with the double burden of hypertension and other cardiovascular diseases along with infection and malnutrition. Hypertension places an excessive financial burden on population and health systems, consuming scarce resources. Population-based preventive approaches are, thus, central for the management of hypertension in developing countries, where clinic-based care for complication is not feasible option. ${ }^{9}$

Furthermore, nearly one-third of the people generally are not aware of having hypertension that makes it an iceberg disease and makes it more difficult to address the whole scenario of the country. The epidemiological studies and surveys on BP can be beneficial in understanding the risk factors that are involved in the progressive rise of BP in our country and can help in uncovering a portion of in-apparent, submerged population at risk of developing hypertension.

In Bangladesh, most people live in villages and most of them are of a low socioeconomic condition. There are many remote villages, where the health care services do not reach. So, the health status of the people in these areas is less known. In case of an emergency condition such as stroke or heart failure, they do not get service near to hand, which lead to death in most of the cases. An elevated BP may be the cause of high mortality among them. So, a proper assessment of the status of hypertension and its risk factors among the rural population in Bangladesh is important to develop strategies and policies for effective prevention and control. The current topic may contribute to lend a hand in gathering the knowledge and information about its status amongst the population of rural areas of Bangladesh and therefore make the health service seekers as well as the planners to be more aware of this deadly non-communicable disease. The objective of the present study was to estimate the proportion of hypertension in an adult population of a selected rural area in Keraniganj Upazila, Dhaka, Bangladesh. 
Materials and Method

The cross-sectional descriptive type of study was conducted in a rural setting of Keraniganj Upazila- in a village named "Malancha" purposively sampling the male and female adult population aged 20 years and above. Data from 199 respondents were collected by face-to-face interview with a pre-tested semi-structured questionnaire, visiting houses in the selected village area. Sphygmomanometer and measuring tape were used as tools for data collection. In case of BP measurement minimum, two measurements were obtained with an interval of at least 1 minute. Then the average BP was used. In case of waist circumference, measurement data were recorded to the nearest $0.1 \mathrm{~cm}$, using a constant tension tape, directly over the skin, at the level of the midpoint between the inferior margin of the last rib and the iliac crest in the mid-auxiliary line. The descriptive analysis was done by a calculator and the results were presented in tables and figures by using a computer.

\section{Results}

Table 1 shows the socio-demographic characteristics of the respondents. In the study, $27.1 \%$ belonged to age group $\geq 30-<40$ years, $26.1 \%$ belonged to age group $<30$ years that was the majority (about $53.2 \%$ ) of the respondents were below the age of 40 years. Most of the respondents were female (53.8\%), Muslims (82.4\%), had primary education $(26.0 \%)$ whereas $19.0 \%$ and $23.0 \%$ of the respondents were illiterate and could sign only, nearly half of the respondents $(47.0 \%)$ were housewives, $85.4 \%$ of the respondents were married and more than half of the respondents $(55.7 \%)$ had monthly income $\geq 10000-<20000$ Taka.

Fig. 1 demonstrates the distribution of the respondents according to their BP status. Of the 199 respondents, 31 (15.6\%) were hypertensive and 168 (84.4\%) normal.

Fig. 2 shows the distribution of hypertension according to age groups. Percentage of hypertension in the age group $\geq 70$ years was found to be the lowest $(6.5 \%)$ and that in $\geq$
$50-<60$ years was found to be the highest (29.0\%).

Table 2 illustrates the hypertension status associated with different risk factors of the respondents. Out of the 199 respondents in the study, $22.6 \%$ took extra fatty food for $3-5$ days per week and among them $22.2 \%$ were hypertensive. Regarding extra salt intake in the study, $55.8 \%$ took extra salt in their food and among them $18.0 \%$ cases were hypertensive. In the study regarding smoking habit, $25.6 \%$ of the respondents were smokers and among them $15.7 \%$ were hypertensive. Furthermore, $36.2 \%$ had a positive family history of hypertension and among them, $19.4 \%$ developed hypertension. Among the respondents who were diabetic $(9.0 \%)$, one-third of them (33.3\%) hypertensive. Moreover, in the study, persons having waist circumference within $100 \mathrm{~cm}-109 \mathrm{~cm}$ were found mostly hypertensive and it was $36.8 \%$ and the minimum number of hypertensive cases were within $60 \mathrm{~cm}-69 \mathrm{~cm}$ of waist circumference and it was $5.6 \%$.

Table 3 describes the distribution of the respondents by the time of being identified as cases of hypertension or hypertensive. Among the respondents, the total known hypertensive cases were $8.6 \%$, newly identified hypertensive cases $7.0 \%$ and nonhypertensive $84.4 \%$.

\section{Discussion}

Countries with an aging population in developed countries will be expected to have a higher prevalence of hypertension than developing countries with a younger population such as Bangladesh, India, etc. However, there are studies, which have documented a high prevalence rate of hypertension in developing countries. ${ }^{9}$

In the study, $27.1 \%$ belonged to age group $\geq$ $30-<40$ years, $26.1 \%$ belonged to age group $<30$ years that was the majority (about $53.2 \%$ ) of the respondents were below the age of 40 years. Most of the respondents were female $(53.8 \%)$, Muslims $(82.4 \%)$, had primary education (26.0\%) whereas $19.0 \%$ and $23.0 \%$ of the respondents were illiterate 
Table 1. Sociodemographic characteristics of the respondents, $n=199$

\begin{tabular}{|c|c|c|}
\hline Characteristics & $\mathbf{n}$ & $\%$ \\
\hline \multicolumn{3}{|c|}{ Age of the respondents (years) } \\
\hline$<30$ & 52 & 26.1 \\
\hline$\geq 30-<40$ & 54 & 27.1 \\
\hline$\geq 40-<50$ & 32 & 16.1 \\
\hline$\geq 50-<60$ & 26 & 13.1 \\
\hline$\geq 60-<70$ & 17 & 8.5 \\
\hline$\geq 70$ & 18 & 9.0 \\
\hline \multicolumn{3}{|c|}{ Sex of the respondents } \\
\hline Male & 92 & 46.2 \\
\hline Female & 107 & 53.8 \\
\hline \multicolumn{3}{|c|}{ Religion of the respondents } \\
\hline Islam & 164 & 82.4 \\
\hline Hindu & 35 & 17.6 \\
\hline \multicolumn{3}{|c|}{ Education of the respondents } \\
\hline Illiterate & 38 & 19.0 \\
\hline Only sign & 46 & 23.0 \\
\hline Primary & 51 & 26.0 \\
\hline Secondary & 38 & 19.0 \\
\hline Higher secondary & 11 & 5.5 \\
\hline Above & 15 & 7.5 \\
\hline \multicolumn{3}{|c|}{ Occupation of the respondents } \\
\hline Service & 47 & 23.0 \\
\hline Business & 40 & 20.0 \\
\hline Student & 11 & 5.5 \\
\hline Housewife & 93 & 47.0 \\
\hline No work & 10 & 5.5 \\
\hline \multicolumn{3}{|c|}{ Monthly Family income, Taka } \\
\hline$<10000$ & 49 & 24.6 \\
\hline$\geq 10000-<20000$ & 111 & 55.7 \\
\hline$\geq 20000-<30000$ & 23 & 11.5 \\
\hline$\geq 30000-<40000$ & 8 & 4.1 \\
\hline$\geq 40000$ & 8 & 4.1 \\
\hline \multicolumn{3}{|l|}{ Marital status } \\
\hline Unmarried & 14 & 7.0 \\
\hline Married & 170 & 85.4 \\
\hline Divorced & 2 & 1.0 \\
\hline Widow & 13 & 6.5 \\
\hline Unmarried & 14 & 7.0 \\
\hline
\end{tabular}




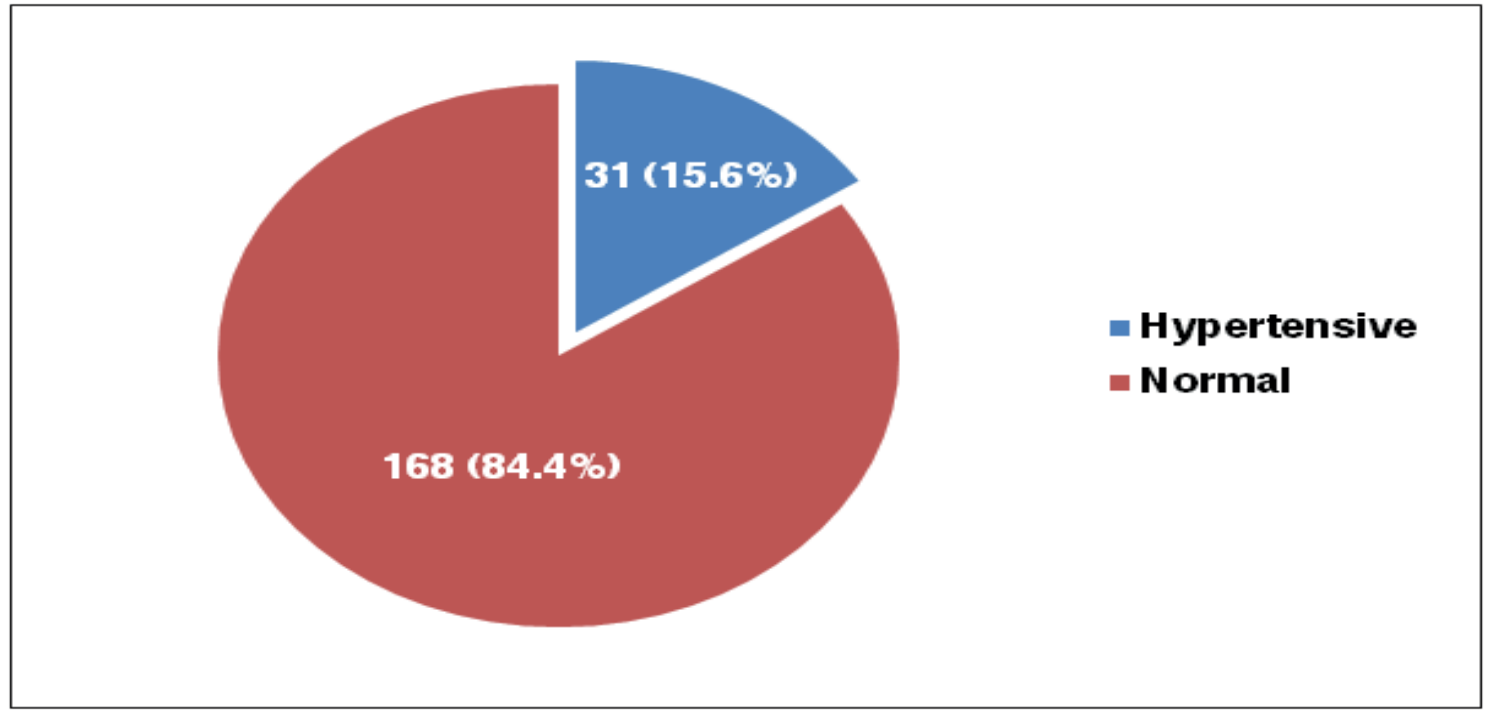

Fig. 1. Distribution of the respondents according to their BP status, $\mathbf{n}=199$.

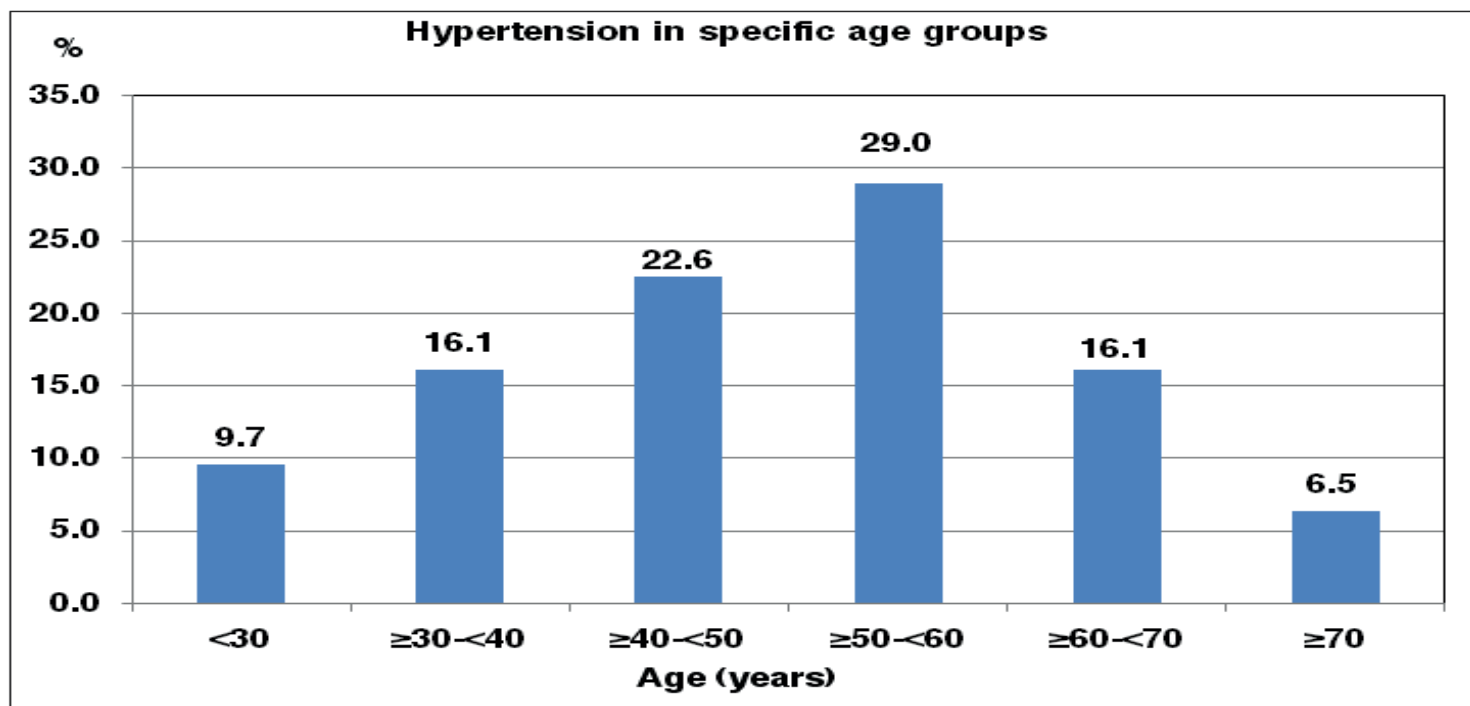

Fig. 2. Distribution of the hypertensive cases according to age groups, $\mathbf{n}=31$.

and could sign only, nearly half of the respondents $(47.0 \%)$ were housewives, $85.4 \%$ of the respondents were married and more than half of the respondents $(55.7 \%)$ had monthly income $\geq 10000-<20000$ Taka.

Now coming to the main issue, in the current study, of the total population, $15.6 \%$ of the respondents were found hypertensive and majority of the population (84.4\%) were nonhypertensive whereas a study in urban area of Dhaka found that the overall age-adjusted prevalence of hypertension was $23.7 \%$ and a survey in a rural community of Bangladesh found the prevalence rate of hypertension in male and female was $31.5 \%$ and $29.4 \%$, respectively. ${ }^{5,9}$ The various studies estimated a prevalence rate of hypertension in Bangladesh ranging from 16.0-34.0\%. However, these differences are due to different study population, different cut-off marks in determining the level of hypertension and different age groups of the study population. Finding of the current study is lower than the prevalence found in other studies. The probability of such difference may be due to the sample on which data are collected was the residents of the adjacent area to the Upazila Health Complex and the Upazila (sub-urban) near to Dhaka city, so the respondents were much aware of their health status and lifestyle. 
Table 2. Hypertension and different risk factors of the respondents

\begin{tabular}{|c|c|c|c|c|}
\hline \multirow[t]{2}{*}{ Trait } & \multicolumn{2}{|c|}{ Total population, $\mathrm{n}=199$} & \multicolumn{2}{|c|}{ With hypertension, $n=31$} \\
\hline & $\mathbf{n}$ & $\%$ & $\mathbf{n}$ & \%* \\
\hline \multicolumn{5}{|l|}{ Fatty food intake } \\
\hline$<2$ days/week & 139 & 69.9 & 20 & 14.4 \\
\hline 3-5 days/week & 45 & 22.6 & 10 & 22.2 \\
\hline 6-7 days/week & 15 & 7.5 & 1 & 6.7 \\
\hline \multicolumn{5}{|l|}{ Extra salt intake } \\
\hline Consumer & 111 & 55.8 & 20 & 18.0 \\
\hline Non-consumer & 88 & 44.2 & 11 & 12.5 \\
\hline \multicolumn{5}{|l|}{ Smoking status } \\
\hline Smoker & 51 & 25.6 & 8 & 15.7 \\
\hline Non smoker & 148 & 74.4 & 23 & 15.5 \\
\hline \multicolumn{5}{|c|}{ Family history of hypertension } \\
\hline Positive family history & 72 & 36.2 & 14 & 19.4 \\
\hline Negative family history & 127 & 63.8 & 17 & 13.4 \\
\hline \multicolumn{5}{|l|}{ Diabetic status } \\
\hline Diabetic & 18 & 9.0 & 6 & 33.3 \\
\hline Non-diabetic & 181 & 91.0 & 25 & 13.8 \\
\hline \multicolumn{5}{|c|}{ Waist Circumference, $\mathrm{cm}$} \\
\hline$<70$ & 18 & 9.0 & 1 & 5.6 \\
\hline$\geq 70-<80$ & 42 & 21.1 & 5 & 11.9 \\
\hline$\geq 80-<90$ & 56 & 28.1 & 6 & 10.7 \\
\hline$\geq 90-<100$ & 59 & 29.7 & 12 & 20.3 \\
\hline$\geq 100-<110$ & 19 & 9.6 & 7 & 36.8 \\
\hline$\geq 110-<120$ & 4 & 2.0 & 0 & 0.0 \\
\hline$\geq 120$ & 1 & 0.5 & 0 & 0.0 \\
\hline
\end{tabular}

$\mathrm{n}$, number; *, $\%$ of the corresponding part of the total respondents.

Table 3. The respondents by the time of being identified as cases of hypertension or non-hypertensive, $\mathrm{n}=199$

\begin{tabular}{lrr}
\hline Respondents & $\mathbf{n}$ & $\mathbf{\%}$ \\
\hline Known hypertensive cases & 17 & 8.6 \\
Newly identified hypertensive cases & 14 & 7.0 \\
Non-hypertensive respondents & 168 & 84.4 \\
\hline Total & 199 & $\mathbf{1 0 0 . 0}$ \\
\hline
\end{tabular}

n, number. 
The present study revealed that percentage of hypertension in the age group of $\geq 70$ years was the lowest $(6.5 \%)$ and among $\geq$ $50-<60$ years of age was the highest $(29.0 \%)$. The number persons with hypertension increased as gradual increase of age up to $\geq 50-<60$ years and after that, it gradually declined. The reason is not clear. Bangladesh Demographic and Health Survey 2011 also found that age was positively associated with $\mathrm{BP}$ values; $18.0 \%$ of women aged $35-39$ were hypertensive compared with $50.0 \%$ of women aged 70 and older. $^{9}$ These findings are similar to other studies in Bangladesh and India, the older age group in urban community was a significant risk factor for the development of hypertension. ${ }^{5}$

The current study revealed that people who took extra fatty food for 3-5 days per week were more prone to develop hypertension than who took fatty food less than 2 days per week. This finding is consistent with a Bangladeshi study reported the recent change in food habits rich in animal protein pattern was positively associated with the prevalence of hypertension. ${ }^{10}$ In this study, $18.0 \%$ were hypertensive among them who took extra salt and $12.5 \%$ among them who did not. So, it can be said that people who took extra salt in their food may be prone to develop hypertension which is similar to other studies in Bangladesh and India. ${ }^{11,12}$ A study showed that participants who took extra salt had almost 1.5 times higher risk of hypertension. ${ }^{5}$ Another recent study showed that in 2010, globally 1.7 million deaths occurred from cardiovascular causes that were attributed to excess sodium consumption above a reference level of $2.0 \mathrm{gm}$ per day. ${ }^{13}$ There is a need to understand the behavioral concepts of salt consumption, develop culturally appropriate materials and create general awareness about the harmful effects of extra salt consumption in Bangladesh.

The present study found that smokers were at higher risk of developing hypertension than the non-smokers which is similar to other studies in Bangladesh and India. ${ }^{11,12}$ Furthermore, it was shown that the risk of developing hypertension was significantly higher among smokers and smokeless tobacco users. ${ }^{5}$ Another study in Bangladesh found a higher prevalence of smoking and showed an increased risk of coronary heart diseases was approximately four folds than ever smokers. ${ }^{13}$

Furthermore, a recent study found that respondents had a positive family history of hypertension were more prone to develop hypertension than those who did not give any family history of hypertension. It was shown by others that family history of stroke/ cerebrovascular diseases (OR 1.23, 95\% Cl 1.02-2.79) also were at high risk of developing hypertension. ${ }^{5}$

In this study, it was revealed that as many as $9.0 \%$ reported of being diabetic. Among the diabetic $33.3 \%$ were hypertensive and among non-diabetic, $13.8 \%$ were hypertensive. Therefore, those who were diabetic were more prone to develop hypertension than the non-diabetics were. Considering waist circumference as a representation of obesity, the current study revealed that those having waist circumference within $100 \mathrm{~cm}$ $109 \mathrm{~cm}$ were found mostly hypertensive $(36.8 \%)$ and the minimum number of hypertensive cases were within $60 \mathrm{~cm}-69 \mathrm{~cm}$ of waist circumference $(5.6 \%)$. It was seen that with increasing of waist circumference the proportion of hypertensive patients also increased. High waist circumference was a significant risk factor for developing hypertension found in other studies in Bangladesh and India. ${ }^{11,12}$

The present study could find that among the population known hypertensive cases were $8.6 \%$ and newly identified hypertensive case were $7.0 \%$. The known cases of hypertension were similar to the newly identified cases. The newly identified cases are the submerged or hidden part of the diseased population suggests the need for a program for early diagnosis and treatment.

Effective strategies for the prevention of hypertension among the adult population should target high-risk groups and the most important determinants of the condition. 
Results of this study might thus contribute to the development of effective prevention strategies and help safeguard the health of the adult population.

\section{Conclusion and recommendations}

Observed hypertension status in the current study present special challenges for the national health care system, given the large resources consumed by the current approaches. Our study provides not only insights into epidemiological information on hypertension and risk factors, but also evidence for developing a feasible, applicable, and cost-effective hypertension management program within the existing primary health care system.

Still hypertension and cardiovascular disease are major contributors to unknown morbidity. Moreover, the condition seems to continue to rise in Bangladesh. Urgent attention should be paid to increase efforts to prevent this rising burden and in addition to identify the appropriate methods to detect the subclinical state- bottom of the iceberg.

Scaling up a population-wide approach, based on healthy lifestyle promotion campaigns, reducing salt consumption and promoting tobacco control, together with an individual prevention approach, based on opportunistic screening, identification of hypertensive or high-risk individuals by easily measurable risk factors, and treatment with an off-patent, low-price multi-drug regime integrated with conventional existing health care might cost-effectively avert a considerable number of deaths from hypertensionrelated cardiovascular diseases, within reasonable health expenditure is needed.

A hypertension care network should be used as a basic framework to set up new intervention strategies for managing more advanced cardiovascular risk factors as well as cardiovascular diseases or other chronic diseases at the primary health care level in the near future in Bangladesh.

\section{References}

1. Chobanian AV, Bakris GL, Black HR, et al.
The Seventh Report of the Joint National Committee on Prevention, Detection, Evaluation, and Treatment of High Blood Pressure. 2003;42(6):1206-52.

2. World Health Organization (WHO), Health Statistics and Informatics Department Geneva, Switzerland. Global Health Risks Summary Tables. WHO; Geneva, 2009.

3. World Health Organization (WHO), Department of Chronic Diseases and Health Promotion. Global Report: Preventing Chronic Diseases- A Vital Investment. WHO; Geneva, 2005.

4. Pereira M, Lunet N, Azevedo A, Barros $\mathrm{H}$. Differences in prevalence, awareness, treatment and control of hypertension between developing and developed countries. J Hypertens 2009; 27(5):963-75.

5. Islam SMS, Mainuddin AKM, Islam MS, et al. Prevalence of risk factors for hypertension: a cross-sectional study in an urban area of Bangladesh. Glob Cardiol Sci Pract 2015:43.

6. MacGill M. Everything you need to know about hypertension. Available from http://www.medicalnewstoday.com/article s/150109.php (Accessed on 23.3.2018).

7. Rahim MA, Rahman MM, Rahman M, Ahmed F, Chowdhury J, Islam F. The prevalence rate of hypertension in rural population of Bangladesh. J Dhaka National Med Coll Hosp 2012;18(1):12-7.

8. National Institute of Population Research and Training (NIPORT), Mitra and Associates, and ICF International. 2013. Bangladesh Demographic and Health Survey 2011. Dhaka, Bangladesh and Calverton, Maryland, USA: NIPORT, Mitra and Associates, and ICF International.

9. Alamgir A, Akhter S. Hypertension prevalence and related factors in an urban affluent community in Bangladesh. Bang J Med Sci 2005;11(1):22-5.

10.Das SK, Sanyal K, Basu A. Study of urban community survey in India: growing trend of high prevalence of hypertension in a developing country. Int $\mathrm{J}$ Med Sci 2005;2(2):70-8.

11.Islam A, Majumder AA. Hypertension in Bangladesh: a review. Indian Heart J 2012;64(3):319-23. 
12.Mozaffarian D, Fahimi S, Singh GM. Global Sodium consumption and death from cardiovascular causes. N Engl J Med 2014;371(7):624-34.

13.Rahman MA, Zaman MM. Smoking and smokeless tobacco consumption: possible risk factors for coronary heart disease among young patients attending a tertiary care cardiac hospital in Bangladesh. Public Health 122(12):1331-8.

Suggestion for citation of the above:

Farhana A, Shakil M, Khan BU, Laskar MS, Alam A. Hypertension status among the adult population in a rural community of Keraniganj Upazila, Dhaka, Bangladesh. Mediscope 2018;5(2):21-9. 